\title{
Mathematical model of innovative sustainability "green" construction object
}

\author{
Michail Slesarev ${ }^{1 *}$, Evgeniy Pankratov ${ }^{2}$ and Viktor Fedorov ${ }^{1}$ \\ ${ }^{1}$ Moscow State University of Civil Engineering, 129337, Yaroslavskoye Shosse 26, Moscow, Russia \\ ${ }^{2}$ Plekhanov Russian University of Economics, 117997, Stremyanny lane, 36, Moscow, Russia
}

\begin{abstract}
The paper addresses the issue of finding sustainability of "green" innovative processes in interaction between construction activities and the environment. The problem of today's construction science is stated as comprehensive integration and automation of natural and artificial intellects within systems that ensure environmental safety of construction based on innovative sustainability of "green" technologies in the life environment, and "green" innovative products. The suggested solution to the problem should formalize sustainability models and methods for interpretation of optimization mathematical modeling problems respective to problems of environmental-based innovative process management, adapted to construction of "green" objects, "green" construction technologies, "green" innovative materials and structures.
\end{abstract}

\section{Introduction}

For years now, the concept of "smart management" has been widely used in the systems theory; the concept shifts the focus on heuristic (expert-based, adaptive, fuzzy, etc.) correction of formal mathematical description of a control object in order to simulate the life environment's dynamics. The known gamut of science and engineering instruments along with effective regulation on environmental research management fail to sufficiently validate the grounds underlying managerial decisions in environmental safety. Numerous issues of the taxonomy of concepts, methods and means related to environmental safety management remain without attention; this includes issues of rational selection of a scientific approach used to describe the processes of environmental safety management in construction, rational choice of modeling and management approach, issues of concurrent use and integration of the results of environmental monitoring along various vectors.

Priority analysis of the growing construction sector of economy seen through the paradigm of construction organization and innovative sustainability based on the principles of innovative governance helped to work out certain approaches to model building for control systems of construction and environmental safety of the living environment. These include: approaches to mathematical interpretation of optimization models for construction and environmental safety management of objects, and models of their innovative sustainability.

Because methods of calculating optimal programs (or trajectories) are well designed by

*Corresponding author: slesarev@mgsu.ru 
now, to ensure optimal allocation of resources, it is sufficient to have a model of the innovation process - its description in the mathematical language $[1,2]$. To compensate for possible interferences and departures from the script, there must be a feedback-generating mechanism of adjusting innovative actions that keeps the innovation process in its preset path [5].

Green technology is comprehensive engineering knowledge, structured with standards of design, manufacturing and construction. The level of its development depends directly on advances of science and technology, activity of industrial engineers, and public awareness of the environmental principles.

In the construction sector, the purpose of the green standards is to speed up the transition from conventional design and construction of buildings and structures towards sustainable development that adheres to the green principles:

- $\quad$ safety and wholesome favorable conditions of human life;

- $\quad$ curbing negative impacts on the environment;

- $\quad$ taking the interests of future generations into account.

Green standards are designed to regulate a robust approach to building and to assess compliance of life environment technologies with the green principles [4].

Development and enforcement of green construction standards creates incentives for business growth on the basis of technical regulation on the market of innovation technologies and products, it also raises the quality of public life and condition of the environment [3].

The hypothesis of innovation-supported environmentally safe development of an object, for example, a thermal or nuclear power plant, understands interpretation of environment parameters under the influence of the object in the form of homogeneous differential equation describing the trajectory of the system "object - environment" traveling through a multiple-parameter information space in the life environment. The required accuracy of research is ensured by known instruments: mathematical optimization models and mathematical tools of motion stability according to the classical automatic control theory.

A fragment of the information space in arbitrary coordinates (materials, components, energy) needed to build an innovative development path for a thermal and nuclear power plant can be illustrated graphically as represented in Fig. 1.

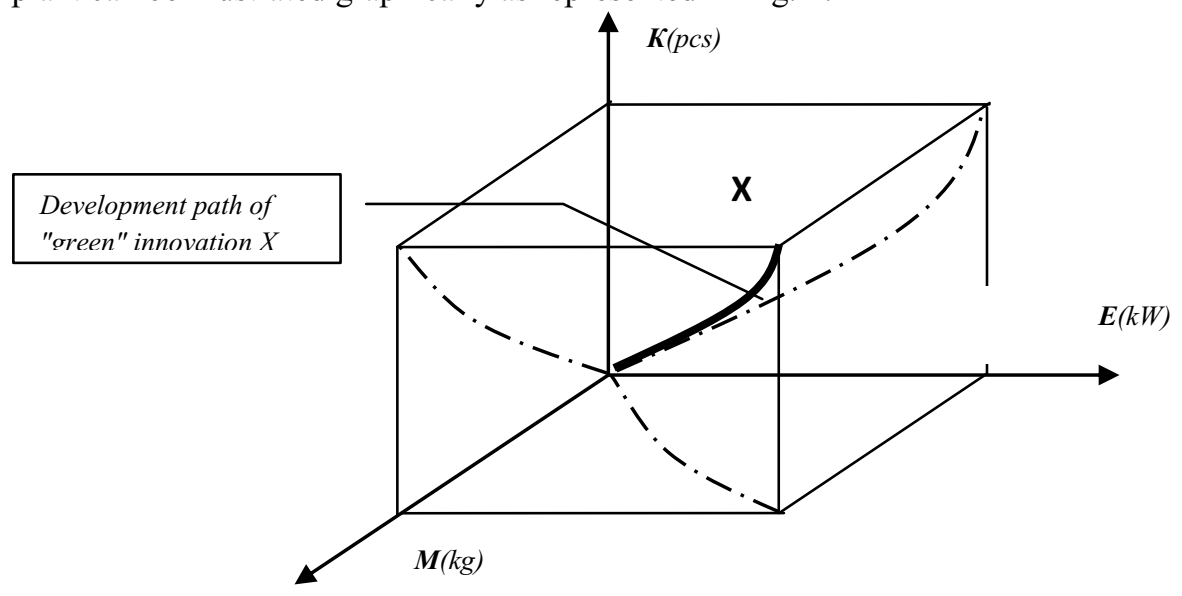

Fig. 1. Graphical interpretation of the development path of an innovative technical object (dynamics of point $\mathrm{X}$ ) in the information coordinates is seen as: $\mathrm{K}$ (number of components in the object); $\mathrm{E}$ (energy spent during the object's life cycle); M (material mass consumed during the object's life cycle). 
To build the development path for a local object of engineering, one has to use data from environmental monitoring parameters of the life environment in the object's particular location.

In general, the equation of the uninhibited development path of a "green" construction within the space of environmental parameters can be represented as:

$$
a_{0} \frac{d^{n} X}{d t^{n}}+a_{1} \frac{d^{n-1} X}{d t^{n-1}}+\cdots+a_{n-1} \frac{d X}{d t}+a_{n} X=0
$$

or symbolically as

where $a_{0}, a_{1}, \ldots a_{n}$ - coefficients of development path, factored by the life environment parameters, and $X$ - relative departure of the control variable of the environment parameter.

As analysis of a system's environmental stability examines unimpeded development of the life environment, the starting conditions of the control system's travel along the development path in the most general case can be represented as follows:

In this case, solution to the homogeneous equation

$$
a_{0} \frac{d^{n} X}{d t^{n}}+a_{1} \frac{d^{n-1} X}{d t^{n-1}}+\cdots a_{n} X=0,
$$

that describes the object's motion through the information space of the life environment parameters, is expressed as:

$$
X(t)=C_{1} e^{p_{1} t}+C_{2} e^{p_{2} t}+\cdots+C_{n} e^{p_{n} t}=\sum_{k=1}^{n} C_{k} e^{p_{k} t},
$$

where $p_{l}, p_{2}, \ldots p_{n}$ - are roots of the characteristic equation

and $C_{1}, C_{2}, \ldots C_{n}-$ are constants determined by the initial conditions.

To ensure that the object in the information space of life environment parameters remains sustainable on terms of innovation, the solution must meet the requirement:

$$
\lim _{t \rightarrow \infty} X(t)=0
$$

This is obviously possible when

$$
\sum_{k=1}^{n} C_{k} e^{p_{k} t} \rightarrow 0
$$

Let us know the restrictions imposed on roots $p_{l}, p_{2}, \ldots p_{n}$, under which the life environment remains ecologically stable.

From among the roots $p$, let us separate those that are real $\alpha_{0}$ from those that are complex $\alpha_{k} \pm$ $j \omega k$. Here, indexes $n$ and $k$ can be different integers.

Now the solution $X(t)$ can be written out as:

$$
X(t)=\sum_{v=1}^{S} C_{v} e^{\alpha_{v} t}+\sum_{k=1}^{n-S} C_{k} e^{\alpha_{v} t} \sin \left(\omega_{k} t+\Psi_{k}\right)
$$


( $S$ - number of real roots).

First we assume that all roots $p$ are simple. Apparently,

$$
\lim _{t \rightarrow \infty} X(t)=0
$$

if each component in the right-hand part tends towards zero. The latter occurs where $\alpha$ $<0$, i.e. the real parts of all roots in the characteristic equation are negative values.

If the real or complex root has the multiplicity of $r$, then the components that form the solution $X(t)$ will have members of the type:

$$
\left.C_{1}^{\prime}+C_{2}^{\prime} t+\cdots+C_{r}^{\prime} t^{r-1}\right) e^{\alpha_{v} t}
$$

and

$$
\left(C_{1}^{\prime \prime}+C_{2}^{\prime \prime} t+\cdots C_{r}^{\prime \prime} t^{r-1}\right) \cdot e^{\alpha_{k} t} \sin \left(\omega_{k} t+\Psi_{k}\right) .
$$

For negative real parts of the roots in a characteristic equation $(\alpha<0)$ the presence of polynomial factors of $t$ will bear no influence on the final result, because $e^{\alpha t}$ at $t \rightarrow \infty$ decreases faster than the polynomial of $t$ increases:

$$
\lim _{t \rightarrow \infty}=\left(C_{1}^{\prime}+C_{2}^{\prime} t+\cdots+C_{r}^{\prime} t^{r-1}\right) e^{\alpha_{\nu} t}=0
$$

Next we consider the case of zero roots and purely imaginary roots when the array of $\alpha$ contains.

Here, there are two ways to address the issue of environmental sustainability. For a simple zero root, the general solution will have a constant component that determines the stationary error.

\section{Conclusion}

If there is at least one root with positive real part among all roots, the "green" technology control system in the life environment and/or "green" innovative products will be unstable.

The prerequisite and sufficient condition for the stability of the control system for innovative technologies and innovative products is that the real parts of roots in the characteristic equation should be negative .

\section{References}

1. V.I. Telichenko, M.Yu. Slesarev, Construction Materials, Equipment and Technologies of XXI Century, 2. 6-7 (1999)

2. M.Yu. Slesarev, Abstract of dissertation for the degree of Doctor of Technical Sciences, 43 (Moscow, FGBOU VO NIU MGSU, 2007)

3. K.Yu. Korolevskiy, M.Yu. Slesarev, Ind. and Civil Constr., 4, $55-57$ (2008)

4. A. Zhukov, Y. Bobrova, D. Zelenshchikov, R. Mustafaev, A. Khimich, Adv, Mat, Res., 1025-1026, 1031-1034 (2014)

5. A. Volkov, A. Sedov, P. Chelyshkov, A. Pavlov, L. Kievskiy, Int. Jour. of App. Eng. Res., 11, 1645-1648 (2016)

6. V.G. Borkovskaya, App. Mech. and Mat., 467, 287-290 (2014) 